\title{
Discovery of Non-Nucleoside Inhibitors of HIV-1 Reverse Transcriptase Competing with the Nucleotide Substrate**
}

\author{
Giovanni Maga, Marco Radi, Samantha Zanoli, Fabrizio Manetti, Reynel Cancio, \\ Ulrich Hübscher, Silvio Spadari, Chiara Falciani, Montserrat Terrazas, Jaume Vilarrasa, and \\ Maurizio Botta*
}

atitle change OK? we avoid the use of words such as new, novel, first, etc in the title $\square$ In the fight against AIDS, first and second generation non-nucleoside reverse transcriptase inhibitors (NNRTIs) are considered essential components of first-line anti-HIV-1 therapy. ${ }^{[1]}$ Structural and biochemical data reveal that, albeit structurally very different, both first and second generation NNRTIs bind to the non-nucleoside inhibitor binding pocket (NNIBP).${ }^{[2]}$ However, although firstgeneration NNRTIs adopt an almost-rigid butterflylike conformation, second-generation NNRTIs are more flexible and show additional contacts with NNIBP. ${ }^{[3]}$ Even if the detailed crystallographic information available for NNRTIs has allowed explanation of both the mechanism of resistance induced by the different mutations and the high level of crossresistance characteristic of this class of compounds, the rapid selection of mutant strains requires the development of new drugs possibly endowed with an alternative mechanism of action.

We have recently reported the development of a straightforward combinatorial approach for the synthesis of 6-vinylpyrimidine derivatives whose structure may be related to that of TNK-651 more than to that of any other known NNRTIs. ${ }^{[4]}$

[*”] Dr. M. Radi, Dr. F. Manetti, Dr. C. Falciani, Prof. M. Botta Dipartimento Farmaco Chimico Tecnologico

University of Siena

Via Alcide de Gasperi 2, 53100 Siena (Italy)

Fax: (+39) 0577-234-333

E-mail: botta@unisi.it

Dr. G. Maga, Dr. S. Zanoli, Dr. R. Cancio, Dr. S. Spadari Istituto di Genetica Molecolare

IGM-CNR

Via Abbiategrasso 207, 27100 Pavia (Italy)

Dr. U. Hübscher

Institut für Veterinärbiochemie und Molekularbiologie

Universität Zürich-Irchel

8092 Zürich (Switzerland)

Dr. M. Terrazas, Prof. J. Vilarrasa

Departament de Química Orgànica

Facultat de Química

Universitat de Barcelona

Av. Diagonal 647, 08028 Barcelona (Spain)

$\left[{ }^{\circ} *\right.$ ] This study was partially supported by grants from the European TRIoH Consortium (LSHB-2003-503480), M.B. thanks Dr. Paolo Malizia for support in the earlier times of his career.

0 Supporting information (including experimental data, docking studies, a color copy of Figure 5, and complete references for this article) is available on the WWW under http://Www.angewandte.org or from the author.
A preliminary biological screening of these compounds on wild-type (wt) HIV-1 reverse transcriptase (RT) allowed the development of a focused library of derivatives, a selection of which (1-5) is described herein (Figure 1 A) ${ }^{[5]}$ Enzymological studies revealed that such compounds bind the NNIBP of the enzyme but, contrary to the NNRTIs reported to date, they inhibit HIV-1 RT by a competitive mechanism with the nucleotide substrate. The most potent analogue, 2-methylsulfonyl-4-dimethylamino-6-vinylpyrimidine (1), is endowed with high activity toward both wt RT and drug-resistant mutants.

To determine the mechanism of inhibition of 6-vinylpyrimidines, they were titrated in reverse-transcription assays in vitro in the presence of various concentrations of either the nucleic acid or the nucleotide substrates. As a result, inhibition exerted by the tested compounds was sensitive to changes in the nucleotide concentration (Figure 1B), resulting in an increase in the apparent $K_{\mathrm{m}}$ for $2^{\prime}$-deoxythymidine5 -triphosphate (dTTP; Figure 1C). On the other hand, no effect on RT inhibition was observed when the nucleic acid concentration was varied (data not shown). These results clearly indicate that the tested 6-vinylpyrimidines 1-5 are competitive inhibitors of RT with respect to the nucleotide substrate (graphical data for compounds $\mathbf{2 , 4 , 5}$ are reported in the Supporting Information). As a comparison, the structurally related 1-[(2-hydroxyethoxy)methyl]-6-(phenylthio)thymine (HEPT) analogue TNK-651 showed a purely noncompetitive mechanism of inhibition with respect to the nucleotide substrate, as shown by the lack of significant variations in the apparent $K_{\mathrm{m}}$ value for dTTP, both with the RT wt and the K103N mutant (see Figure S2 in the Supporting Information). In the absence of co-crystals between RT and our compounds, their binding site was established by testing their sensitivity to known NNRTI-resistant mutations localized in the NNIBP. ${ }^{[6-8]}$ As shown in Table 1, inhibitory activity of the tested compounds was strongly affected by these mutations, which supports the hypothesis that 6-vinylpyrimidines bind to the NNIBP and act therefore as nonclassical competitive inhibitors. Molecular docking and dynamics simulations were finally performed to investigate the interaction mode of these ligands with the NNIBP (both of wt and mutated RT) and to suggest a possible explanation for their unique mechanism of action.

Compound 1 was docked into the wt HIV-1 RT NNIBP (Figure $2 \mathrm{~A}$ ) starting from the X-ray coordinates of the TNK651:HIV-1 RT complex (PDB code: 1RT2) ${ }^{[9]}$ which were chosen on the basis of the similarity between compounds 1-5 
A<smiles>C=Cc1cc(N(C)C)nc(S(C)(=O)=O)n1</smiles><smiles>C=Cc1cc(NCC)nc(S(C)(=O)=O)n1</smiles><smiles>C=Cc1cc(NC(C)C)nc(S(C)(=O)=O)n1</smiles><smiles>C=Cc1nc(SC)nc(N(C)C)c1Cl</smiles><smiles>C=Cc1cc(N(C)C)nc(SCc2ccc(OC)cc2)n1</smiles>

5

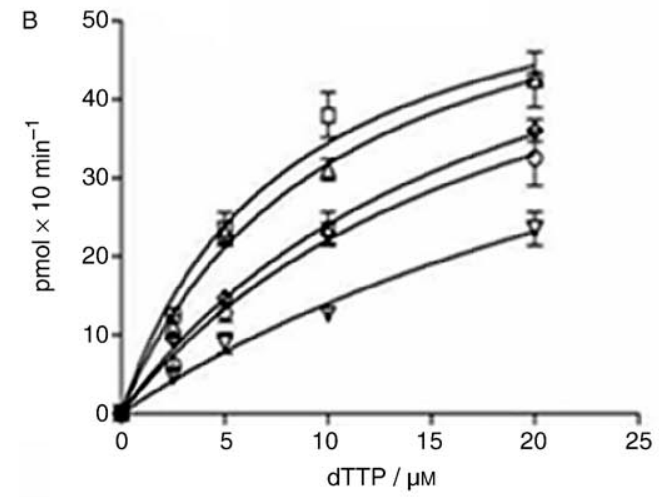

a none

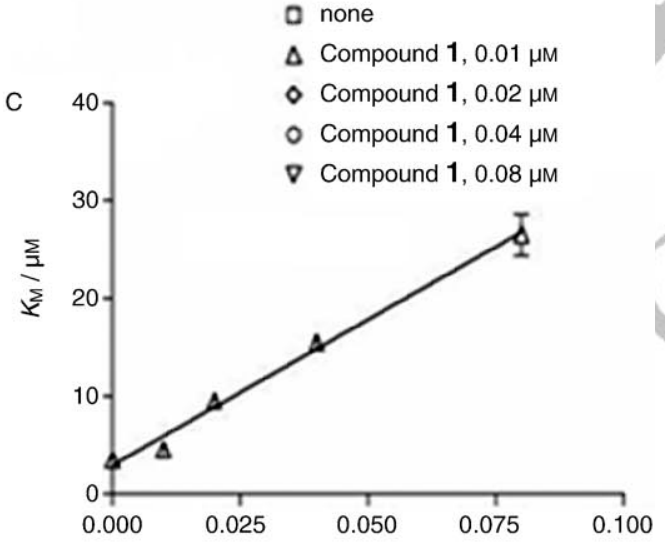

$[1] / \mu \mathrm{M}$

Figure 1. A) 6-Vinylpyrimidine inhibitors $\mathbf{1}-\mathbf{5}$ and the reference compound TNK-651. B) Plot of the incorporation rates of wt HIV-1 RT showing the variation of the reaction rate as a function of the dTTP substrate concentration in the absence or in the presence of increasing amounts of compound 1. Curves were fitted to a Briggs-Haldane mechanism. Error bars represent the standard deviation of three independent replicates. C) Variation of the apparent affinity $\left(K_{m}\right)$ for the nucleotide substrate as a function of the concentration of $1 . K_{m}$ values were determined as described in the Supporting Information from the curves shown in panel $B$.
Table 1: Inhibitory activity of 6-vinylpyrimidines 1-5 toward wt and mutant HIV-1 RT.

\begin{tabular}{|c|c|c|c|c|c|}
\hline \multirow[t]{2}{*}{ compd } & \multicolumn{5}{|c|}{$K_{\mathrm{i}}[\mu \mathrm{M}]^{[a]}$} \\
\hline & wt & K103N & Y181I & L100I & V179D \\
\hline 1 & 0.008 & 3.2 & 39 & 0.012 & 0.015 \\
\hline 2 & 3.5 & $>400$ & $>400$ & n.d..$^{[b]}$ & n.d. \\
\hline 3 & $>400$ & $>400$ & $>400$ & n.d. & n.d. \\
\hline 4 & 90 & $>400$ & $>400$ & n.d. & n.d. \\
\hline 5 & 325 & $>400$ & $>400$ & n.d. & n.d. \\
\hline
\end{tabular}

[a] $\mathrm{K}_{\mathrm{i}}=$ inhibition constant; [b] n.d. = not determined.

and TNK-651. The reliability of the docking protocol was tested on the prediction of the binding geometries of the reference compound TNK-651 into the NNIBP. As a result, the experimental binding conformation of the reference drug was successfully reproduced with acceptable root-meansquare deviation $(0.966 \AA$ ) of atom coordinates (see Figure $2 \mathrm{~B}$ ).

The energetically preferred docked conformation of $\mathbf{1}$ revealed interactions that may contribute to the stability of the resulting inhibitor:RT complex. The heterocyclic ring of the ligand was found at close contact with Tyr188, Tyr181, and Phe 227 (allowing $\pi-\pi$ interactions), whereas the vinyl group interacted with Tyr 318. Moreover, additional profitable hydrophobic contacts between the methyl groups of the amine moiety and Trp229 are noted. The very polar sulfone group is oriented toward the water-exposed surface, in proximity of the positive charge of the Lys101 ammonium group (for further docking studies see the Supporting Information). To shed light on the peculiar mechanism of action of the 6-vinylpyrimidines $\mathbf{1 - 5}$, the progression of the conformational changes in the side chain of Met230, Asp 110, Asp 185, and Asp186 (key residues for the polymerization process) was monitored by means of molecular dynamics (MD) simulations. In this regard, it was clearly seen that, as the simulation progresses, the side chain of Met230 achieves an extended conformation (Figure 3A, white to blue). This new conformation could affect the positioning of the growing viral DNA (forcing the growing nucleic acid chain to reorient) and the subsequent polymerization process. By monitoring the conformational changes of the aspartic acid triad (Figure $3 \mathrm{~A}$, white to blue), it was interesting to note that, as the simulation progresses, the catalytic site opened so that the Asp185 side chain was gradually shifted $5.59 \AA$ away from its initial position ( $t=0$ in the MD simulation). In case of the 2:RT (wt) complex, the catalytic site triad also assumed an open conformation during the MD simulations even if this distortion was smaller than that observed for the most-active compound (1). In fact, the Asp 185 side chain extended only $3.52 \AA$ away from its initial position and this could account for the lower inhibitory activity of 2 (Figure $3 \mathrm{~B}$ ). Whatever their mechanism of inhibition, all the known NNRTIs bind the NNIBP. It is therefore reasonable to expect that a comparison of the crystal structures of non-ligand-bound HIV-1 RT with those of HIV-1 RT complexed with common NNRTIs and with the output of our MD simulations should yield valuable insights into the peculiar mechanism of action of the 6vinylpyrimidines described herein. Superposition analysis 


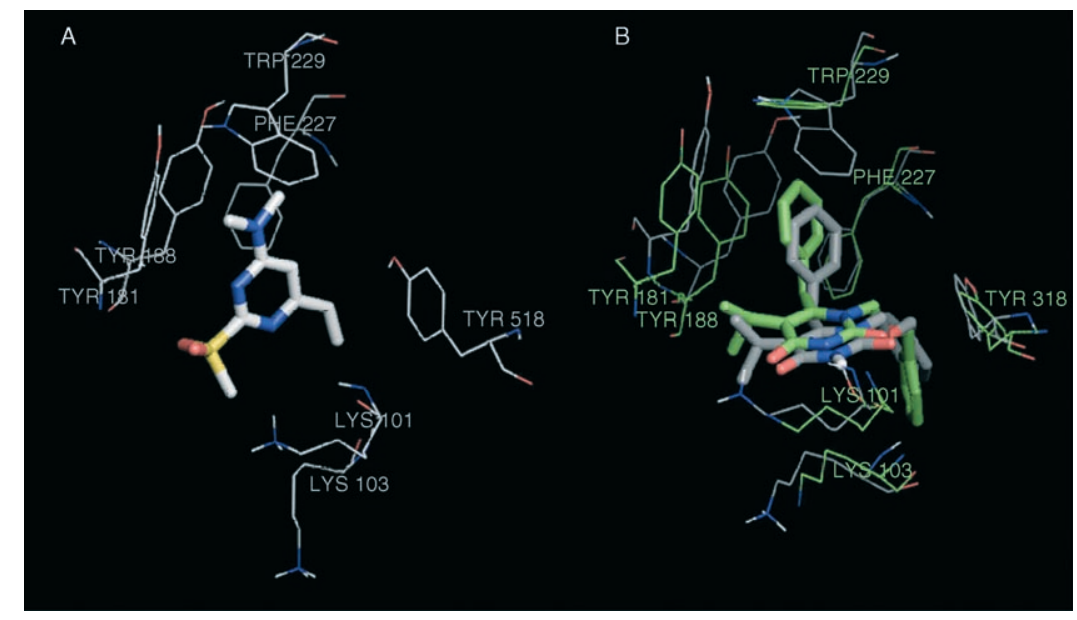

Figure 2. A) Docking of 1 (sticks) into the NNIBP of the TNK-651:HIV-1 RT complex. B) Comparison of the docked TNK-651 (green, sticks) and X-ray conformation of TNK-651 (grey, thick lines) into NNIBP. For reason of clarity, only the side chains of Tyr 181, Tyr 188, Trp 229, Phe 227, Tyr 318, Lys 103, and Lys 101 are represented as thin lines. Please note that Angewandte Chemie has decided to cover the color costs of Figure 2 घ
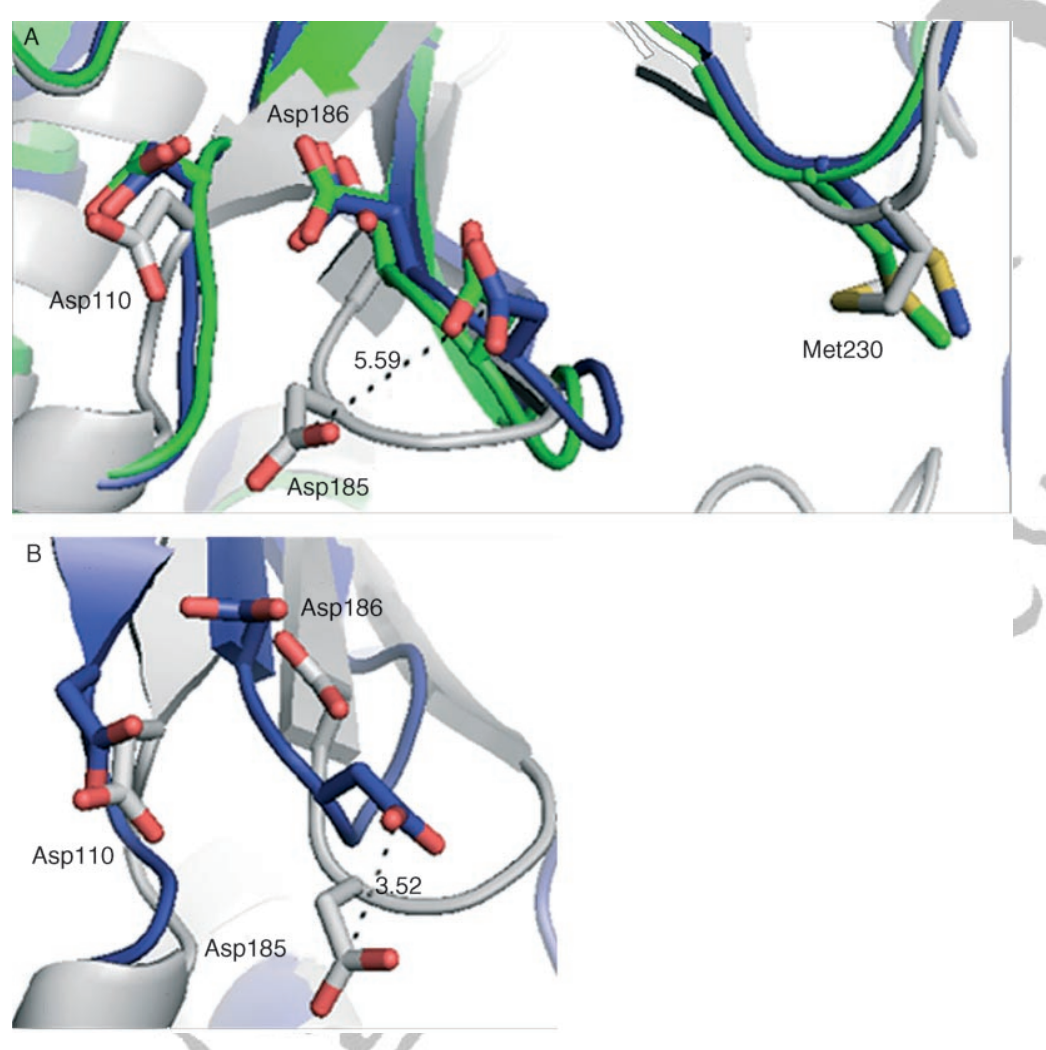

Figure 3. Overlay of representative snapshots from the MD trajectory showing the evolution of the position of relevant RT amino acid residues: A) 1:RT complex, side chains of Met 230, Asp 110, Asp 185, and Asp 186 are shown as sticks. Ribbons and carbon atoms of the side chains of these amino acids are colored according to time frames in the order white $\rightarrow$ green $\rightarrow$ blue $(0,500$, and $1000 \mathrm{ps}$, respectively); B) 2:RT complex, ribbons, and side-chain carbon atoms are colored according to time frames in the order white $\rightarrow$ blue ( 0 and 1000 ps, respectively).

depicted in Figure 4 clearly shows that the binding of common first- and second-generation NNRTIs (TNK-651, ${ }^{[9]}$ efavir- enz ${ }^{[10]}$ nevirapine, ${ }^{[11]}$ and R185545 ${ }^{[12]}$ ) to the NNIBP determines only a major shift of the primer grip (Met230), whereas the aspartic acid triad does not seem to experience substantial modifications compared with the non-ligand-bound enzyme. By contrast, it was interesting to note that although the binding of compound $\mathbf{1}$ to the NNIBP (1:RT complex at $t=1000 \mathrm{ps}$ ) determined only a small change to the side chain of Met 230 (white circle in Figure 4), a significant and peculiar shift was noted for Asp185 (red circle in Figure 4), which was shifted far away from the aspartic acid triad (i.e. 7.66 $\AA$ away from its position in the TNK-651:RT complex). Furthermore, when the same MD simulation was performed on the nonligand-bound RT and the RT:TNK-651 complex, no significant shift for Asp 185 was observed. On the basis of these observations, it is reasonable to argue that the unusual shift of Asp185 could ultimately be responsible for the competitive mechanism of action exerted by the 6-vinylpyrimidines as, when $\mathbf{1}$ is bound to the allosteric site, the magnesium ions in the catalytic site may lie so apart that no phosphodiester bond formation can take place. From a visual inspection of the polymerase active site, we moreover speculated that the conformational rearrangements responsible for the competitive mechanism of action may originate from the disruption of the typical type II geometry of the $\beta$ turn formed by the conserved Tyr-Met-Asp-Asp sequence (residues 183-186), which are responsible for the correct positioning of the aspartate residues of the catalytic site (Figure 5). ${ }^{[13]}$

In fact, it is well known that the formation of a hydrogen bond between Gln 182 and Met 184 is required for the stabilization of the otherwise strained type II conformation of this turn in the wt RT (Figure 5 A). In a similar way, the complex RT:TNK-651 retained the Gln182:Met 184 hydrogen bond and, consequently, displayed the same strained type II geometry of the $\beta$ turn (Figure $5 \mathrm{C}$ ). On the contrary, in the 1:RT complex, a conformational rearrangement of the $\beta$ turn occurred so that the side chains of Asp 185 and Glu 182 were shifted away from their original position (Figure $5 \mathrm{~B}$ ). As a consequence, the unfavorable steric interaction between the $C \beta$ atom of Met184 and the amide group of Asp 185 disappeared and the hydrogen bond between Gln 182 and Met 184 was lost. This result further supports the peculiar behavior of compound $\mathbf{1}$, which is able to induce conformational modifications otherwise not found in complexes between RT and common NNRTIs. In summary, the present work reports the identification of a new class of

These are not the final page numbers! 


\section{Communications}

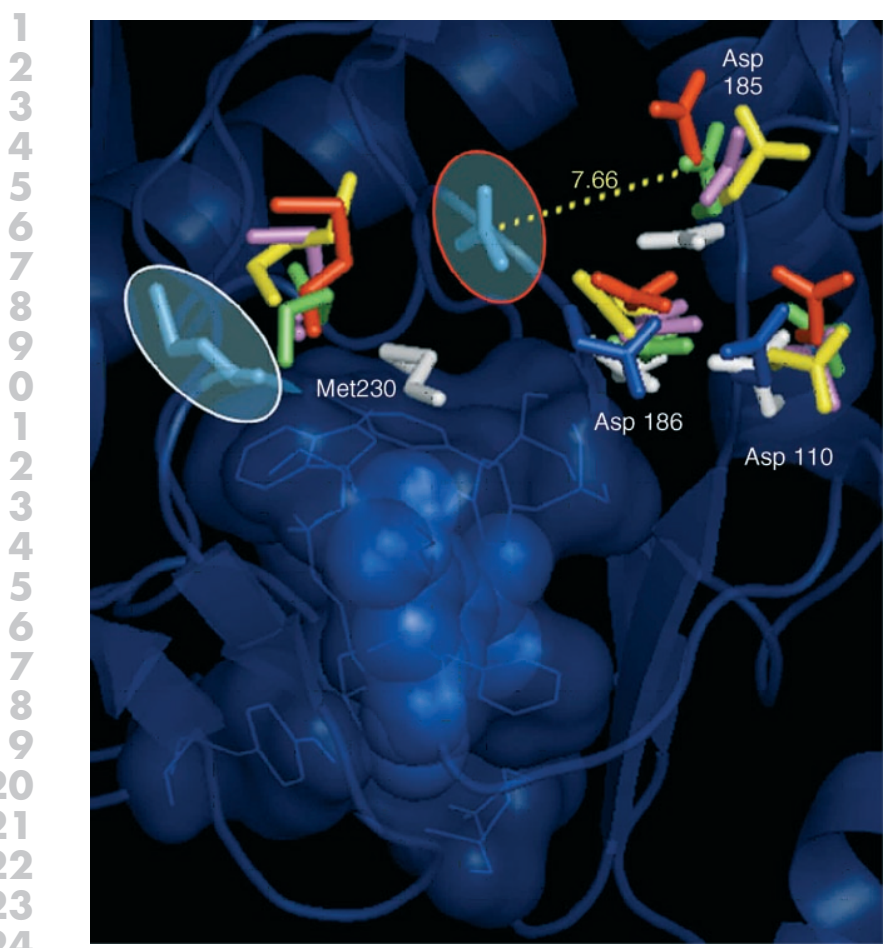

Figure 4. Superimposition of the structures corresponding to the 1:RT complex (blue) at $t=1000 \mathrm{ps}$, TNK-651:RT complex (green; PDB code: 1RT2), efavirenz:RT complex (violet; PDB code: 1IKW), nevirapine:RT complex (yellow; PDB code: 3HVT), R185545:RT complex (red; PDB code: 1SUQ), unliganded RT (white; PDB code: 1HMV). White and red circles evidence conformational rearrangements of Met230 and Asp 185 (respectively) for the 1:RT complex in comparison with the other inhibitor:RT complexes. For reason of clarity, only the fundamental residues (Met 230, Asp 110, Asp 185, and Asp 186) of non-ligandbound RT and RT complexed with reference inhibitors are shown as sticks. Compound 1 bound to the NNIBP is shown as blue spheres.

NNRTIs with a 6-vinylpyrimidine scaffold found to exhibit a peculiar behavior: contrary to the NNRTIs reported to date, enzymological studies reveal that such compounds inhibit HIV-1 RT by a competitive mechanism with the nucleotide substrate after binding to the NNIBP of the enzyme. To the best of our knowledge, these compounds represent the first example of NNRTIs found to exhibit such a behavior.

Received: October 11, 2006

Revised: December 19, 2006

Published online: $\mathbf{\square} \mathbf{\square}, 2007$

Keywords: competitive inhibitors · drug design · enzymes · HIV1 reverse transcriptase pyrimidines

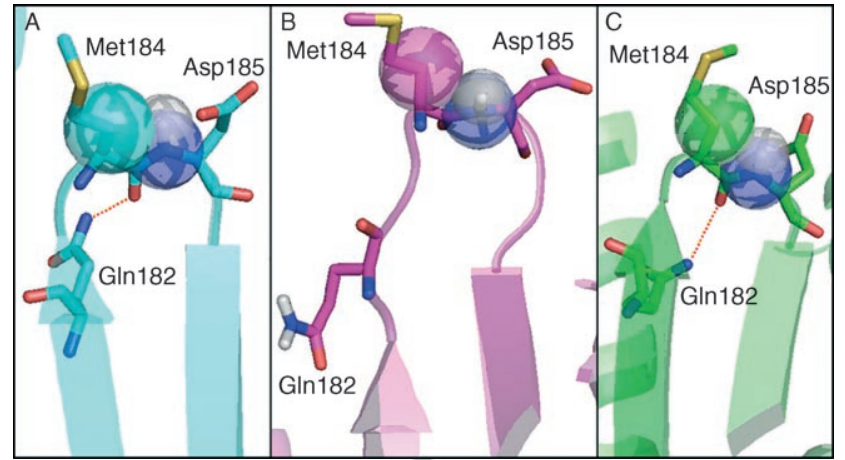

Figure 5. Structure of the $\beta$ turn (ribbons) around the active site of RT. Steric interactions between $C \beta$ atom of Met184 and the amide group of Asp185 (represented as transparent spheres) and hydrogen bonds (dotted lines) can be appreciated. A) non-ligand-bound RT (PDB code: $1 \mathrm{HMV}$ ); $\mathrm{B}$ ) 1:RT complex (at $\mathrm{t}=1000 \mathrm{ps)}$ ) C) TNK-651:RT complex (PDB code: 1RT2). All figures produced with Pymol. ${ }^{[14]}$

[1] G. Barbaro, G. Barbarini, Chemotherapy 2006, 52, 161-165.

[2] J. Ding, K. Das, H. Moereels, L. Koymans, K. Andries, P. A. Janssen, S. H. Hughes, E. Arnold, Nat. Struct. Biol. 1995, 2, $407-$ 415.

[3] K. Das, P. J. Lewi, S. H. Hughes, E. Arnold, Prog. Biophys. Mol. Biol. 2005, 88, 209-231.

[4] M. Radi, E. Petricci, G. Maga, F. Corelli, M. Botta, J. Comb. Chem. 2005, 7, 117-122.

[5] M. Botta, F. Corelli, E. Petricci, M. Radi, G. Maga, Italian Patent RM2005A000512 ם, year?

[6] E. De Clercq $\square$ please insert other authors $\square \mathbf{\square}$, Virology 1993, 192, 246-253.

[7] J. W. Mellors, G. E. Dutschman, G. J. Im, E. Tramontano, S. R. Winkler, Y. C. Cheng, Mol. Pharmacol. 1992, 41, 446-451.

[8] J. H. Nunberg, W. A. Schleif, E. J. Boots, J. A. O’Brien, J. C. Quintero, J. M. Hoffman, E. A. Emini, M. E. Goldman, J. Virol. 1991, 65, 4887-4892.

[9] D. I. Stuart $\square$ please insert other authors $\square$, J. Med. Chem. 1996, 39, 1589-1600.

[10] J. Lindberg, S. Sigurdsson, S. Lowgren, H. O. Andersson, C. Sahlberg, R. Noreen, K. Fridborg, H. Zhang, T. Unge, Eur. J. Biochem. 2002, 269, 1670-1677.

[11] J. Wang, S. J. Smerdon, J. Jager, L. A. Kohlstaedt, P. A. Rice, J. M. Friedman, T. A. Steitz, Proc. Natl. Acad. Sci. USA 1994, 91, $7242-7246$.

[12] K. Das घplease insert other authors $\square$, J. Med. Chem. 2004, $47,2550-2560$.

[13] D. W. Rodgers, S. J. Gamblin, B. A. Harris, S. Ray, J. S. Culp, B. Hellmig, D. J. Woolf, C. Debouck, S. C. Harrison, Proc. Natl. Acad. Sci. USA 1995, 92, 1222-1226.

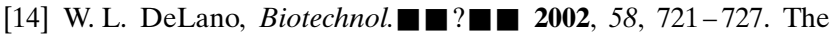
PyMOL Molecular Graphics System, DeLano Scientific, San Carlos, CA. 


\section{Communications}

\section{Competitive Inhibitors}

G. Maga, M. Radi, S. Zanoli, F. Manetti,

R. Cancio, U. Hübscher, S. Spadari,

C. Falciani, M. Terrazas, J. Vilarrasa,

M. Botta*

III-III

Discovery of Non-Nucleoside Inhibitors of HIV-1 Reverse Transcriptase

Competing with the Nucleotide Substrate

Kompetitive Inhibitoren

G. Maga, M. Radi, S. Zanoli, F. Manetti,

R. Cancio, U. Hübscher, S. Spadari,

C. Falciani, M. Terrazas, J. Vilarrasa,

M. Botta*

III-III

Discovery of Non-Nucleoside Inhibitors of HIV-1 Reverse Transcriptase

Competing with the Nucleotide Substrate

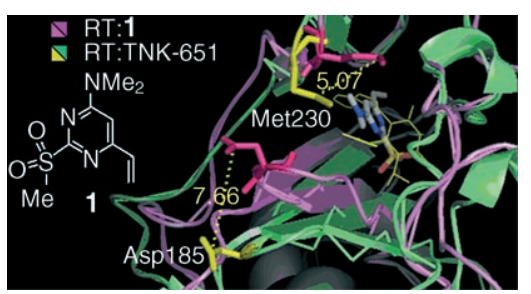

How odd! A new class of non-nucleoside reverse transcriptase inhibitors with a 6 vinylpyrimidine scaffold (1) has been found to inhibit HIV-1 reverse transcriptase (RT) by a competitive mechanism with the nucleotide substrate after binding to the non-nucleoside inhibitors binding pocket of the enzyme. Molecular modeling studies have been performed to elucidate their peculiar behavior.

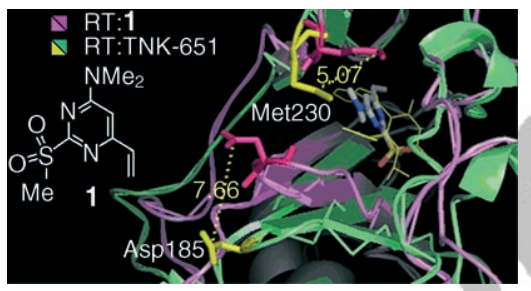

Ungewöhnlich! Eine neue Klasse von Nichtnucleosid-Reverse-TranskriptaseInhibitoren mit einem 6-Vinylpyrimidingerüst (1) inhibiert die reverse HIV-1Transkriptase (RT) durch Konkurrenz mit dem Nucleotidsubstrat nach der Bindung an die Enzym-Bindetasche für Nichtnucleosid-Inhibitoren. Mithilfe von Molecular Modeling wurde versucht, dieses ungewöhnliche Verhalten zu erklären. 